\title{
Sulforaphane Attenuation of Type 2 Diabetes-Induced Aortic Damage Was Associated with the Upregulation of Nrf2 Expression and Function
}

\author{
Yonggang Wang, ${ }^{1,2}$ Zhiguo Zhang, ${ }^{1,2}$ Wanqing Sun, ${ }^{1}$ Yi Tan, ${ }^{2,3,4}$ Yucheng Liu, ${ }^{2}$ Yang Zheng, \\ Quan Liu, ${ }^{1}$ Lu Cai, ${ }^{2,3,4}$ and Jian Sun ${ }^{1}$ \\ ${ }^{1}$ Cardiovascular Center at the First Hospital of Jilin University, 71 Xinmin Street, Changchun 130021, China \\ ${ }^{2}$ Kosair Children's Hospital Research Institute, Department of Pediatrics, University of Louisville, Louisville, KY 40202, USA \\ ${ }^{3}$ The Chinese-American Research Institute, Wenzhou Medical University, Wenzhou 325035, China \\ ${ }^{4}$ Departments of Radiation Oncology, Pharmacology and Toxicology, University of Louisville, Louisville, KY 40292, USA
}

Correspondence should be addressed to Lu Cai; 10cai001@louisville.edu and Jian Sun; sunjianemail@126.com

Received 3 November 2013; Revised 11 December 2013; Accepted 6 January 2014; Published 23 February 2014

Academic Editor: Adriane Belló-Klein

Copyright (C) 2014 Yonggang Wang et al. This is an open access article distributed under the Creative Commons Attribution License, which permits unrestricted use, distribution, and reproduction in any medium, provided the original work is properly cited.

\begin{abstract}
Type 2 diabetes mellitus (T2DM) significantly increases risk for vascular complications. Diabetes-induced aorta pathological changes are predominantly attributed to oxidative stress. Nuclear factor E2-related factor-2 (Nrf2) is a transcription factor orchestrating antioxidant and cytoprotective responses to oxidative stress. Sulforaphane protects against oxidative damage by increasing Nrf2 expression and its downstream target genes. Here we explored the protective effect of sulforaphane on T2DM-induced aortic pathogenic changes in C57BL/6J mice which were fed with high-fat diet for 3 months, followed by a treatment with streptozotocin at $100 \mathrm{mg} / \mathrm{kg}$ body weight. Diabetic and nondiabetic mice were randomly divided into groups with and without 4-month sulforaphane treatment. Aorta of T2DM mice exhibited significant increases in the wall thickness and structural derangement, along with significant increases in fibrosis (connective tissue growth factor and transforming growth factor), inflammation (tumor necrosis factor- $\alpha$ and vascular cell adhesion molecule 1), oxidative/nitrative stress (3-nitrotyrosine and 4-hydroxy-2-nonenal), apoptosis, and cell proliferation. However, these pathological changes were significantly attenuated by sulforaphane treatment that was associated with a significant upregulation of $\mathrm{Nrf} 2$ expression and function. These results suggest that sulforaphane is able to upregulate aortic Nrf2 expression and function and to protect the aorta from T2DM-induced pathological changes.
\end{abstract}

\section{Introduction}

Type 2 diabetes mellitus (T2DM) is a growing public health problem, associated with a substantial burden of morbidity and mortality [1]. Youth with T2DM had higher rates of all complications than nondiabetes subjects, and an overall 6.15fold increased risk of any vascular disease [2]. Individuals with T2DM have a unique propensity towards microvascular and macrovascular diseases [3]. The macrovascular disorders include atherosclerosis, coronary artery disease, and peripheral vascular diseases. Although application of drugs and changing life style had been widely promoted to control the complications, unfortunately, the preventive of the development and progression of vascular complications in the diabetic patients remains unoptimistic [4]. Therefore, an effective approach to prevent and/or delay the development and progression of diabetic vascular complications urgently needs to be developed.

Impaired endothelial function is considered as a major diabetic vascular alteration, which may be mainly derived from diabetes-induced overexpression of inflammation. Diabetic inflammation in the vascular endothelium leads to continuous infiltration and accumulation of leukocytes at sites of endothelial cell injury. Consistent with our previous studies, vascular inflammatory response was increased in diabetic mice reflect by increased expression of tumor necrosis factor-alpha (TNF- $\alpha$ ) and vascular cell adhesion molecule 1 (VCAM-1) [5, 6]. It is known that inflammation and oxidative 


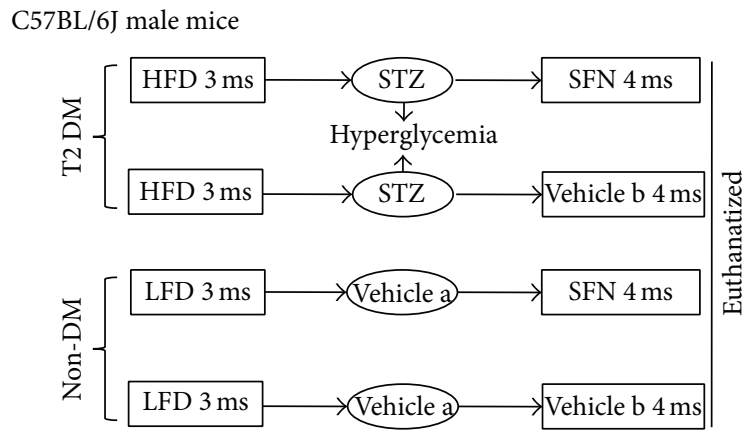

FIGURE 1: Schematic illustration for the animal experimental design.

stress are reciprocal cause and outcomes [7]. There was also increasing evidence indicating that the increased production of reactive oxygen and/or nitrogen species (ROS and/or RNS) is the major pathogenic factor responsible for the development and progression of vascular complications in diabetic patients, although several other mechanisms are also proposed [8-10]. Clinical trials with single antioxidants or a few have shown ineffective intervention in diabetic patients $[6,9-11]$; therefore, upregulation of multiple endogenous antioxidants may be a better approach for the prevention of diabetic vascular complications. Transcription factor nuclear factor E2-related factor-2 (Nrf2) has been shown to play a pivotal role in cellular preventing against oxidative stress and damage in vitro and in vivo $[12,13]$. Under physiological conditions, Nrf2 generally localizes in the cytoplasm and binds to its inhibitor Kelch-like ECH-associated protein 1 (Keap1) [14]; however, under oxidative or electrophilic stress conditions, Nrf2 detaches from Keapl and translocates into the nucleus to bind to antioxidant-responsive elements (ARE) in the promoter region of its downstream genes, such as NADPH quinoneoxidoreductase (NQO1), heme oxygenase-1 (HO-1), glutathione S-transferase (GST), superoxide dismutase (SOD), catalase (CAT), and other genes regulating the responses to oxidative stress $[15,16]$. The Nrf2-ARE pathway is important in the cellular detoxification, antioxidant, and anti-inflammatory system to protect the cell and tissue from oxidative stress $[17,18]$. Therefore, Nrf2 is widely appreciated for its potential prevention of and/or therapy for diabetic vascular complications $[5,19]$.

Sulforaphane (SFN) is an isothiocyanate that is found in cruciferous vegetables and has a strong cytoprotective function against oxidative stress. This beneficial effect was mediated by its direct interaction with Keapl, resulting in the disruption of Nrf2-Keap1 interaction. Released Nrf2 from Keap1 enters into the nucleus to induce the expression of Nrf2 downstream antioxidant genes [14]. Evidence has confirmed the specific cysteine residues of Keapl that act as "sensors" to be modified by SFN [20]. SFN as a Nrf2 activator [21] has been reported to prevent oxidative damage [22] and cardiovascular diseases [23]. SFN has garnered particular interests as an indirect antioxidant due to its extraordinary ability to induce expression of endogenous, multiple enzymes via the upregulation of Nrf2 function [24]. Our previous study has found that SFN had a beneficial effect on type 1 diabetic vascular complications [5]. However, there was no report yet whether SFN can prevent the development of aortic pathogenesis alterations in T2DM.

To this end, we used a type 2 diabetic mouse model to verify the protective function of SFN against diabetic aortic damage and dissect the underlying mechanisms. The experimental design was illustrated in Figure 1. Type 2 diabetic and age-matched control mice were treated with SFN for 4 months. At the end of 4 months treatment of SFN mice were euthanatized for collecting tissues to perform the experimental measurements.

\section{Materials and Methods}

2.1. Animals. C57BL/6J male mice, 8-10 weeks of age, were purchased from the Jackson Laboratory (Bar Harbor, Maine) and housed at the University of Louisville Research Resources Center at $22^{\circ} \mathrm{C}$ with a $12 \mathrm{~h}$ light/dark cycle with free access to food and tap water. All experimental procedures for these animals were approved by the Institutional Animal Care and Use Committee of the University of Louisville, which is compliant with the Guide for the Care and Use of Laboratory Animals published by the US National Institutes of Health (NIH Publication number 85-23, revised 1996).

2.2. Type 2 Diabetes Model. To establish type 2 diabetic model, mice were fed with high-fat diet (HFD, Research Diets 12492, 60\% kcal from fat) for 3 months, which made these mice become significantly obese $(44.6 \pm 2.9 \mathrm{~g}$ for HFD versus $32.4 \pm 1.5 \mathrm{~g}$ for control, $P<0.05)$. Insulin resistance was also induced, shown by the increased area size under curves for both Intraperitoneal (IP) glucose tolerance test (IPGTT) (35493.28 \pm 5270.90 for HFD versus $25114.82 \pm$ 4630.55 for control, $P<0.05)$ and IP insulin tolerance test (IPITT) $(15403.26 \pm 3252.76$ for HFD versus $9790.5 \pm 3462.36$ for control, $P<0.05)$. These insulin resistant mice were randomly injected intraperitoneally with STZ (Sigma-Aldich, St. Louis, MO, dissolved in $0.1 \mathrm{M}$ sodium citrate ( $\mathrm{pH} 4.5$, Vehicle a, Figure 1)) at $100 \mathrm{mg} / \mathrm{kg}$ body weight once [25, 26]. Five days after STZ injection, mice with hyperglycemia (blood glucose levels $\geq 250 \mathrm{mg} / \mathrm{dL}$, blood sample collected from the tail vein measured using a Free Style Lite glucometer (Abbott Diabetes Care, Alameda, CA)) were defined as diabetic. In parallel, age-matched control mice were given low-fat diet (LFD, Research Diets 12450B, 10\% kcal from fat) for 3 month, followed by an injection of the same volume of sodium citrate buffer when HFD-fed mice were received STZ injection. Both diabetic and control mice continually received HFD or LFD feeding for additional 4 months (Figure 1). During this 4-month period, both diabetic and control mice were further divided into two groups, with and without SFN treatment (Sigma-Aldich), which were given SFN at $0.5 \mathrm{mg} / \mathrm{kg}$ subcutaneously for five days per week. Dose of SFN used was based on our previous study [27].

At the end of the additional 4 months, these diabetic mice remained showing the significantly increased insulin resistance $(29230 \pm 3173.43$ for HFD versus $7947.50 \pm 1209.02$ for control, $P<0.05)$ and blood glucose $(324.66 \pm 51.07$ for HFD versus $116.00 \pm 11.04$ for control, $P<0.05$ ), insulin 

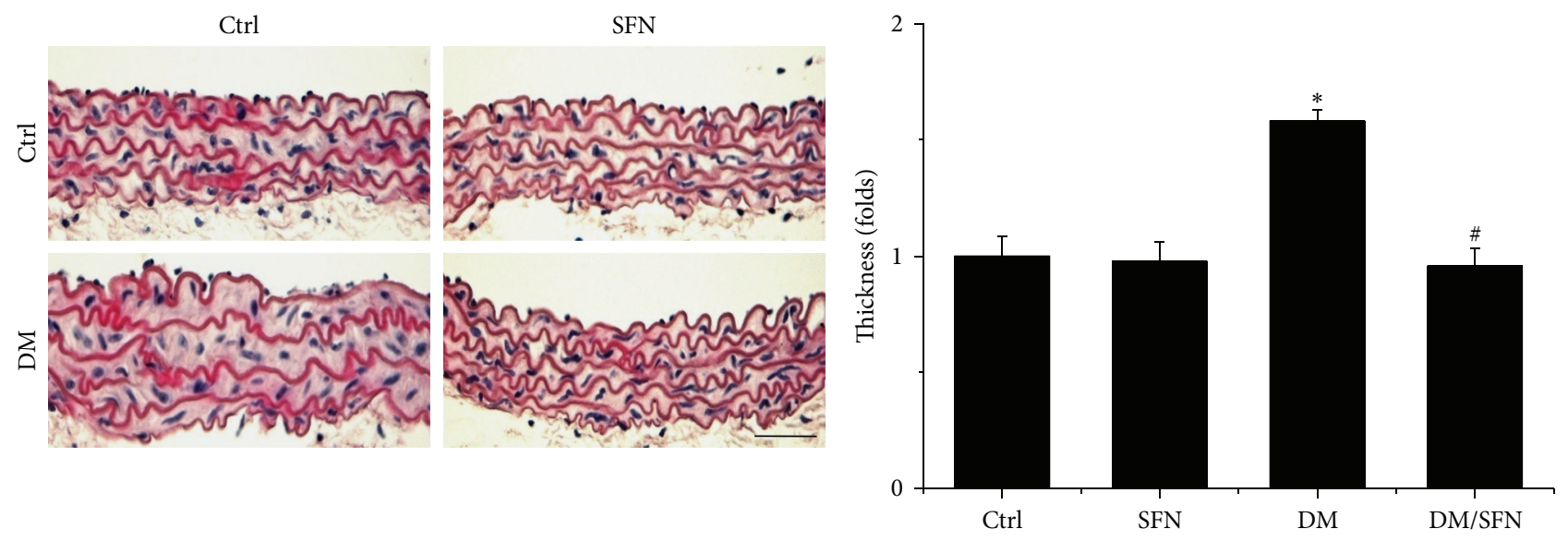

(a)
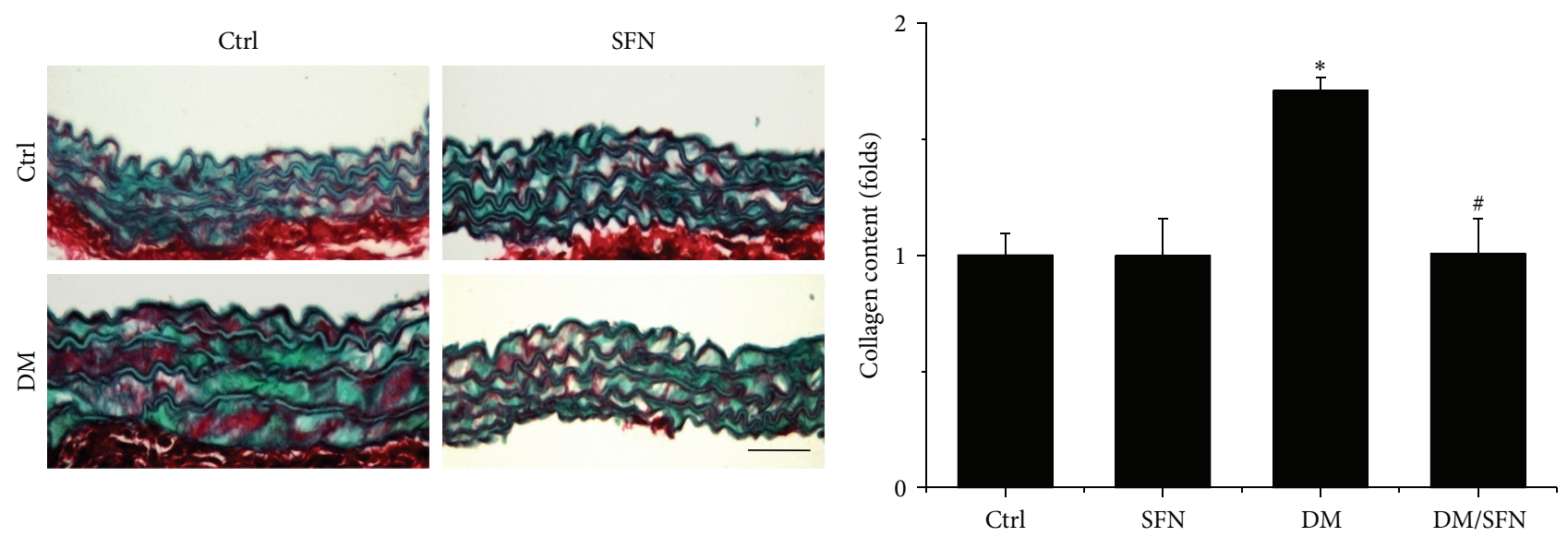

(b)

FIgURE 2: Protective effect of SFN on diabetes-induced aortic pathological changes. The pathogenic changes of aortas were examined by H\&E staining (a) and the accumulation of collagen was detected by Sirius-red staining (b), followed by semiquantitative analysis. Data were presented as means $\pm \mathrm{SD}(n=6) ;{ }^{*} P<0.05$ versus corresponding Ctrl; ${ }^{\#} P<0.05$ versus corresponding DM. Bar $=50 \mu \mathrm{M}$.

$(0.8293 \pm 0.13$ for HFD versus $0.55 \pm 0.05$ for control, $P<$ $0.05)$, triglyceride $(395.05 \pm 64.43$ for HFD versus $48.48 \pm 4.80$ for control, $P<0.05)$, and cholesterol (139.12 \pm 6.53 for HFD versus $80.39 \pm 15.56$ for control, $P<0.05)$ levels; all of which are typical magnifications, suggesting the induction of T2DM. In summary, there were four groups of mice $(n=6$ at least per group): LFD control (Ctrl), LFD/SFN (SFN), type 2 diabetes (DM), and DM plus SFN (DM/SFN). At the end of 4 months of SFN treatment, mice were euthanatized for experimental measurements. Since SFN was dissolved in $1 \%$ dimethyl sulfoxide (DMSO) and diluted in PBS, mice serving as controls were also subcutaneously given the same volume of PBS containing 1\% DMSO (Vehicle b, Figure 1), based on our own and other studies [27-29].

\subsection{Aorta Preparation and Histopathological Examination.} After mice were anesthetized with 2,2,2-tribromoethanol (commercial name: avertin) at $4-6 \mathrm{mg} / \mathrm{kg}$ body weight, thoraxes were opened and the descending thoracic aortas were isolated carefully without rips or cuts. Aortic tissues were fixed in $10 \%$ buffered formalin overnight. The fixed tissues were cut into ringed segments (approx. 2-3 $\mathrm{mm}$ in length) so they can be dehydrated in graded alcohol series, clean with xylene, embedded in paraffin, and sectioned at $5 \mu \mathrm{m}$ thickness for pathological and immunohistochemical or immunofluorescent staining. Histological evaluation of aorta was performed by $\mathrm{H} \& \mathrm{E}$ staining. The thickness of aorta was evaluated by measuring the width of tunica media using Image Pro Plus 6.0 software. For immunohistochemical or immunofluorescent staining, paraffin sections from aortic tissues were dewaxed, incubated with 1x Target Retrieval Solution (Dako, Carpinteria, CA) in a microwave oven for $15 \mathrm{~min}$ at $98^{\circ} \mathrm{C}$ for antigen retrieval, followed by $3 \%$ hydrogen peroxide for $10 \mathrm{~min}$ at room temperature and $5 \%$ bovine serum albumin for $60 \mathrm{~min}$, respectively. These sections were then incubated with primary antibodies against connective tissue growth factor (CTGF) at 1:100 dilution (BD Bioscience, San Jose, CA) and transforming growth factor (TGF- $\beta 1$ ) at 1:100 dilution (Santa Cruz Biotechnology, Santa Cruz, CA, USA), tumor necrosis factor-alpha (TNF- $\alpha$ ) at 1:50 dilution (Abcam, Cambridge, MA), vascular cell adhesion molecule 1 (VCAM-1) at 1:100 dilution (Santa Cruz 


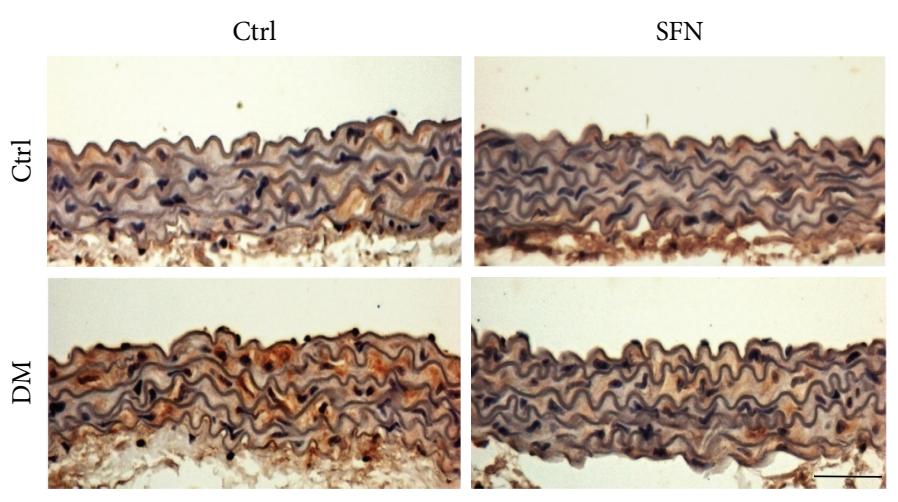

(a)

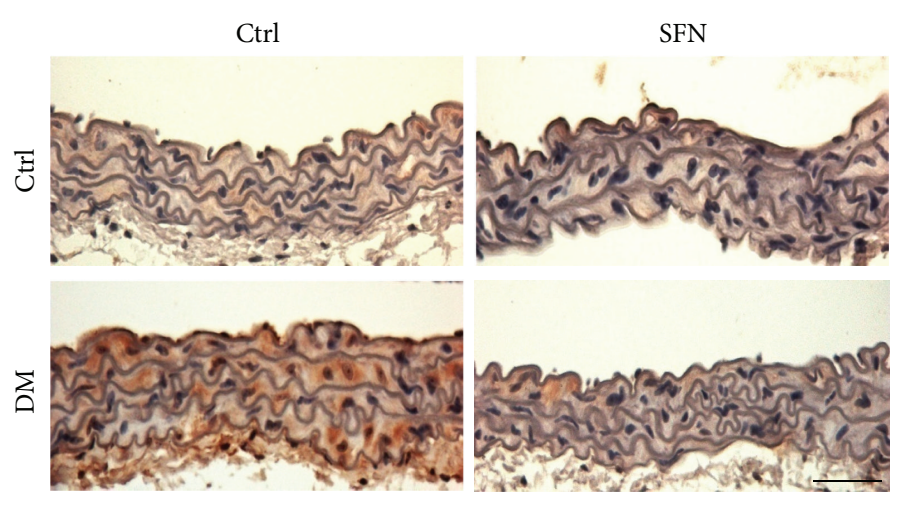

(b)
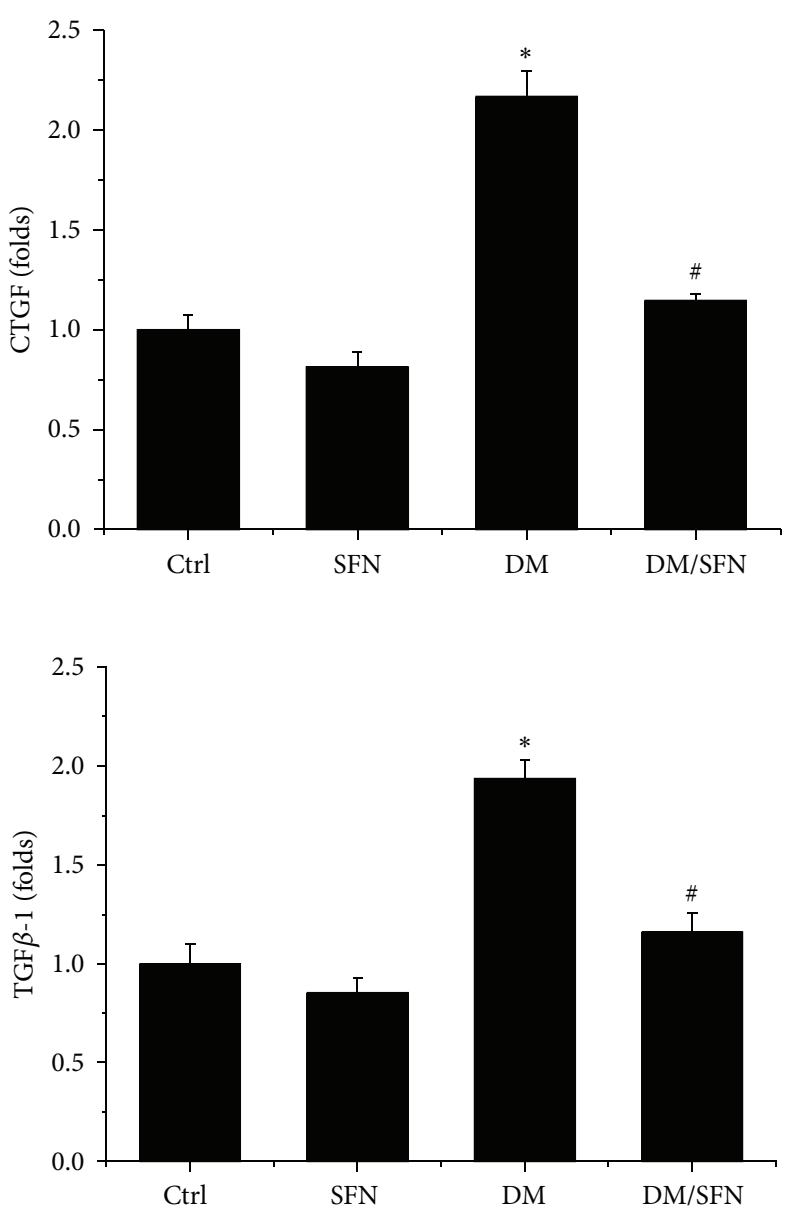

FIGURE 3: Protective effect of SFN on diabetes-induced aortic fibrosis. Aortic fibrosis was examined by immunohistochemical staining for the expression of CTGF (a) and TGF- $\beta 1$ (b), followed by semiquantitative analysis. Data were presented as means \pm SD $(n=6)$; ${ }^{*} P<0.05$ versus corresponding Ctrl; ${ }^{\#} P<0.05$ versus corresponding DM. Bar $=50 \mu \mathrm{M}$.

Biotechnology, Santa Cruz, CA, USA), 3-nitrotyrosine (3NT) at 1:400 dilution (Millipore, Billerica, CA), 4-hydroxy2-nonenal (4-HNE) at 1:400 dilution (Alpha Diagnostic International, San Antonio, TX), Nrf2 at 1:50 dilution, and $\mathrm{Cu}-\mathrm{Zn}$ super oxide dismutase-1 (SOD-1) at 1:400 dilution (both from Santa Cruz Biotechnology, Santa Cruz, CA, USA) over night at $4^{\circ} \mathrm{C}$. Afterwards sections were washed with PBS, and incubated with horseradish peroxidase conjugated secondary antibody (1:100-400 dilutions with PBS) or Cy3-coupled donkey anti-rabbit or anti-goat IgG secondary antibody (1:200 dilution with PBS) for $1 \mathrm{~h}$ at room temperature. For color development purposes, immunohistochemical staining sections were treated with peroxidase substrate DAB kit (Vector Laboratories, Inc. Burlingame, CA) and counterstained the nuclei with hematoxylin, while immunofluorescent staining sections were stained with DAPI at 1:1000 dilution to localize the nucleus.

For quantitative analysis of these immunohistochemical and immunofluorescent staining, the Nikon Eclipse E600 microscopy system was used and 3 sections at interval of 10 sections from each aorta (per mouse) were selected and at least five high-power fields randomly sections were randomly recaptured. Image Pro Plus 6.0 software was used to translate the interesting area staining density into an integrated optical density (IOD) that was divided by the area size of interest to reflect the staining intensity, and the ratio of IOD/area size in experimental group was presented as a fold relative to that of control.

2.4. Sirius-Red Staining for Collagen. Aortic fibrosis was detected by Sirius-red staining of collagen, as described in our previous study [6]. Briefly sections were stained with $0.1 \%$ Sirius-red F3BA and $0.25 \%$ Fast Green FCF. The stained sections were then assessed for the presence of collagen using a Nikon Eclipse E600 microscopy system.

2.5. Terminal Deoxynucleotidyl-Transferase-Mediated dUTP Nick-End Labeling (TUNEL) Staining. TUNEL staining was performed with formalin-fixed, paraffin-embedded sections using peroxidase in situ Apoptosis Detection Kit S7100 (Millipore, Billerica, MA), according to the manufacture's instruction. The positively stained apoptotic cells were counted randomly in five microscopic fields at least for each of the three slides from each mouse under light microscopy. The 

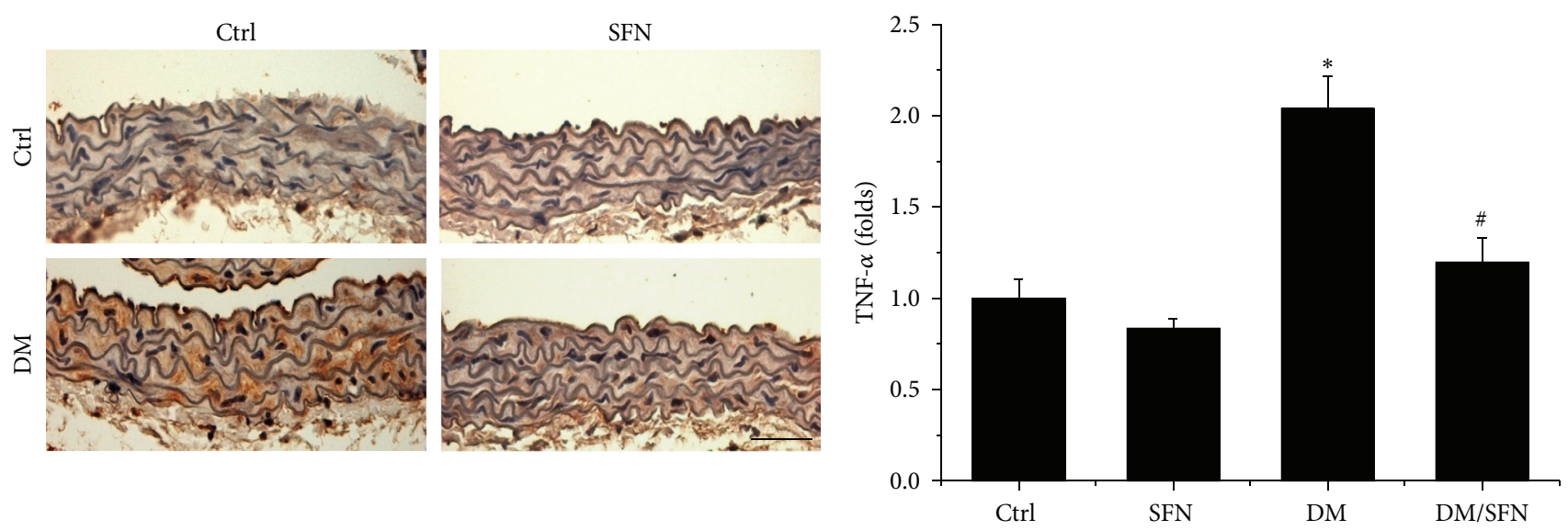

(a)
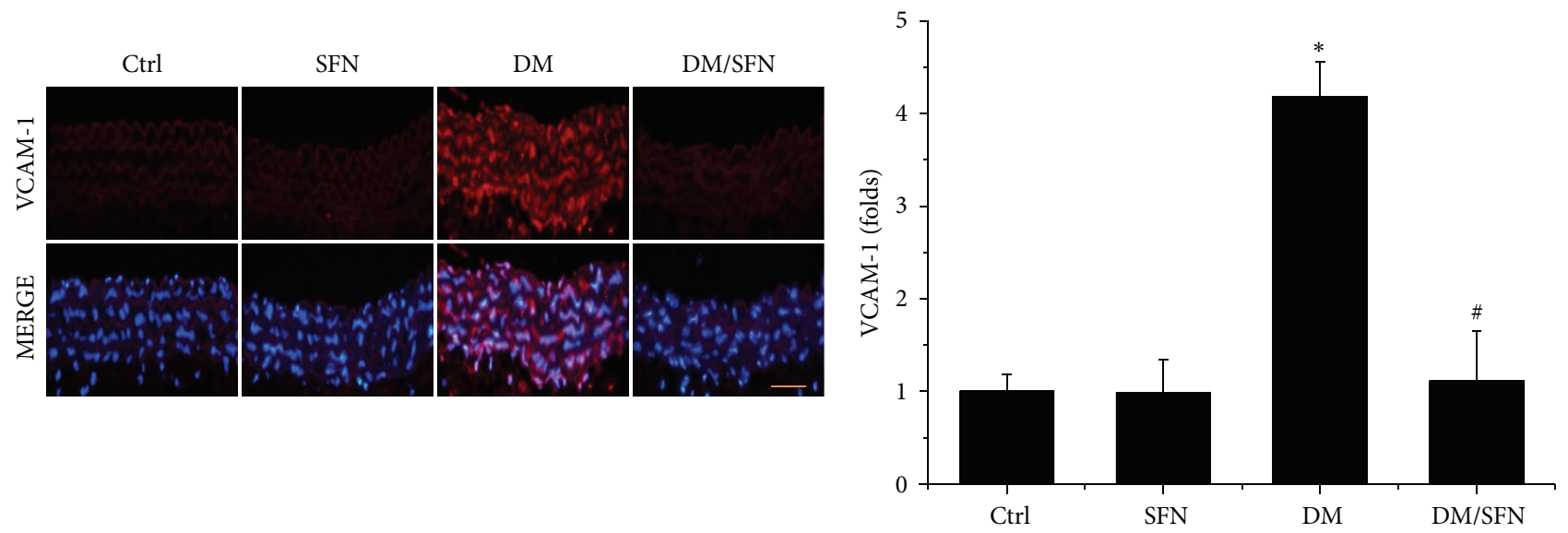

(b)

FIgURE 4: Protective effect of SFN on diabetes-induced aortic inflammation. Aortic inflammation was examined by immunohistochemical staining for the expression of TNF- $\alpha$ (a) and immunofluorescent staining for the expression of VCAM-1 (red) (b), followed by semiquantitative analysis. Data were presented as means $\pm \mathrm{SD}(n=6) ;{ }^{*} \mathrm{P}<0.05$ versus corresponding $\mathrm{Ctrl} ;{ }^{\#} \mathrm{P}<0.05$ versus corresponding DM. Bar $=50 \mu \mathrm{M}$.

percentage of TUNEL positive cells relative to 100 nuclei was presented.

2.6. Proliferating Cell Nuclear Antigen (PCNA) Staining. The PCNA staining kit (Invitrogen, Camarillo, CA) was used for staining proliferating cells, according to the manufacture's instruction. The positively stained proliferating cells were counted randomly in five microscopic fields at least for each of the three slides per mouse under light microscopy. The percentage of PCNA positive cells relative to 100 nuclei was presented.

2.7. Real-Time qPCR. Aortas were frozen with liquid nitrogen and stored at $-80^{\circ} \mathrm{C}$. Total RNA was extracted using the TRIzol Reagent (Invitrogen, USA). RNA concentrations and purity were quantified using a Nanodrop ND-1000 spectrophotometer. First-strand complimentary DNA (cDNA) was synthesized from total RNA according to manufacturer's protocol from the RNA PCR kit (Promega, Madison, WI). Reverse transcription was performed using $0.5 \mu \mathrm{g}$ of total RNA in $12.5 \mu \mathrm{L}$ of the solution containing $4 \mu \mathrm{L} 25 \mathrm{mM} \mathrm{MgCl}_{2}$,
$4 \mu \mathrm{L}$ AMV reverse transcriptase $5 \mathrm{x}$ buffer, $2 \mu \mathrm{L}$ dNTP, $0.5 \mu \mathrm{L}$ RNase inhibitor, $1 \mu \mathrm{L}$ of AMV reverse transcriptase, and $1 \mu \mathrm{L}$ of oligodT primer, which were added with nucleasefree water to make a final volume of $20 \mu \mathrm{L}$. Reaction system was run at $42^{\circ} \mathrm{C}$ for $50 \mathrm{~min}$ and $95^{\circ} \mathrm{C}$ for $5 \mathrm{~min}$. Primers of Nrf2, SOD-1, HO-1, and GAPDH were purchased from Applied Biosystems (Carlsbad, CA). Real-time quantitative PCR (qPCR) was carried out in a $20 \mu \mathrm{L}$ reaction buffer that included $10 \mu \mathrm{L}$ of TaqMan Universal PCR Master Mix, $1 \mu \mathrm{L}$ of primer, and $9 \mu \mathrm{L}$ of cDNA with the ABI 7300 Real-Time PCR system. The fluorescence intensity of each sample was measured at each temperature change to monitor amplification of the target gene. The comparative cycle time (CT) was used to determine fold differences between samples.

2.8. Statistical Analysis. Data were presented as mean $\pm \mathrm{SD}$ $(n=6)$. Comparisons were performed by two-way ANOVA for the different groups. When there was significant difference among groups, the repetitive comparing Tukey's test was used to further analyse with Origin 7.5 Lab data analysis and 


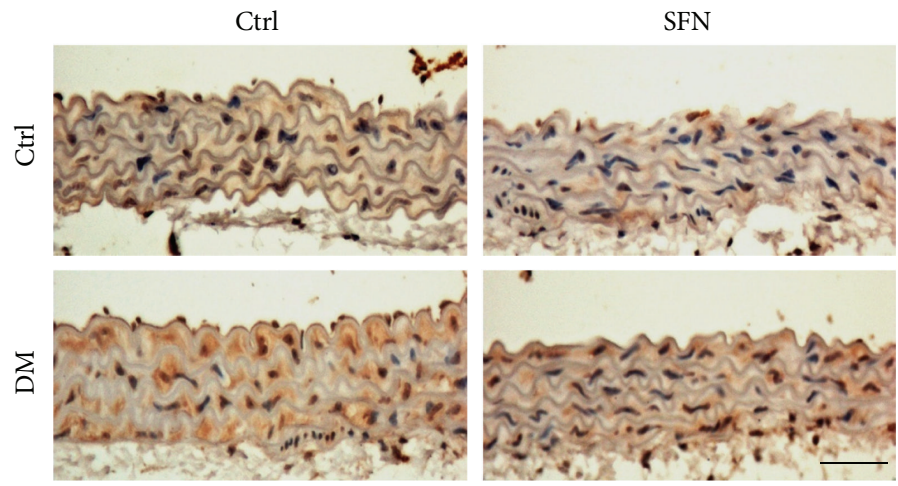

(a)
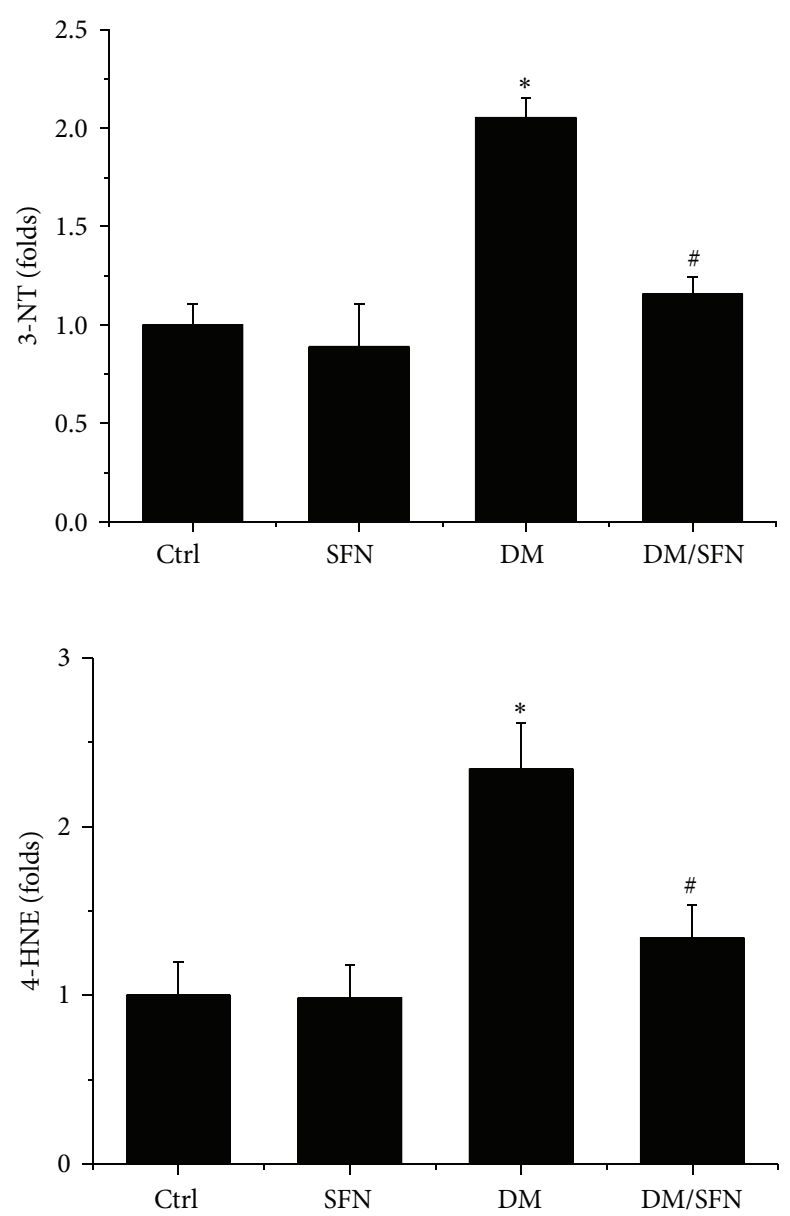

(b)

FIGURE 5: Protective effect of SFN on diabetes-induced aortic oxidative damage. Aortic oxidative damage was examined by immunohistochemical staining for the accumulation of 3-NT (a) and 4-HNE (b), followed by semiquantitative analysis. Data were presented as means \pm $\mathrm{SD}(n=6) ;{ }^{*} P<0.05$ versus corresponding Ctrl; ${ }^{\#} P<0.05$ versus corresponding DM. Bar $=50 \mu \mathrm{M}$.

graphing software. $P<0.05$ was considered as statistical significance.

\section{Result}

3.1. SFN Prevented T2DM-Induced Aortic Pathological Changes and Fibrosis. At the end of experiment, aortas were examined pathologically by $\mathrm{H} \& \mathrm{E}$ staining, which displayed significantly increase of the tunica media thickness in T2DM mice (Figure 2(a)). Sirius-red staining also revealed an increased collagen accumulation in tunica media of aortas in T2DM group (Figure 2(b)). However, all these pathological changes observed in the aortas of T2DM mice were completely prevented by the 4-month SFN treatment.

To further detect the preventive effect of SFN on T2DM-induced aortic fibrosis, immunohistochemical staining showed the increased expression of profibrotic mediators, CTGF (Figure 3(a)), and TGF- $\beta 1$ (Figure 3(b)), in aortic tunica media of diabetic mice. Supplementation with SFN completely prevented these fibrotic responses in the aortas of type 2 diabetic plus SFN mice (DM/SFN group).
3.2. SFN Prevented T2DM-Induced Aortic Inflammation and Oxidative Damage. On account of the fact that both inflammation and oxidative damage are primary risk factors for the vascular endothelium remodeling, the expression of TNF$\alpha$ (Figure 4(a)) and VCAM-1 (Figure 4(b)) was examined with immunohistochemical and immunofluorescent staining, which showed a significant increase in aortic tunica media of T2DM mice, an effect that was completely prevented by 4-month SFN treatment.

Considering that inflammation and oxidative stress are reciprocal cause and outcomes, oxidative and nitrative damage was examined by immunohistochemical staining for increased accumulation of 3-NT (Figure 5(a)) and 4-HNE (Figure 5(b)), which was found to be significantly increased in the aortic tunica media of T2DM mice. However, treatment with SFN for 4 months completely prevented the oxidative damage (Figures 5(a) and 5(b)).

3.3. SFN Prevented T2DM-Induced Aortic Apoptotic Cell Death and Proliferation. We recently reported the induction of apoptotic cell death and cell proliferation in the aortas of 


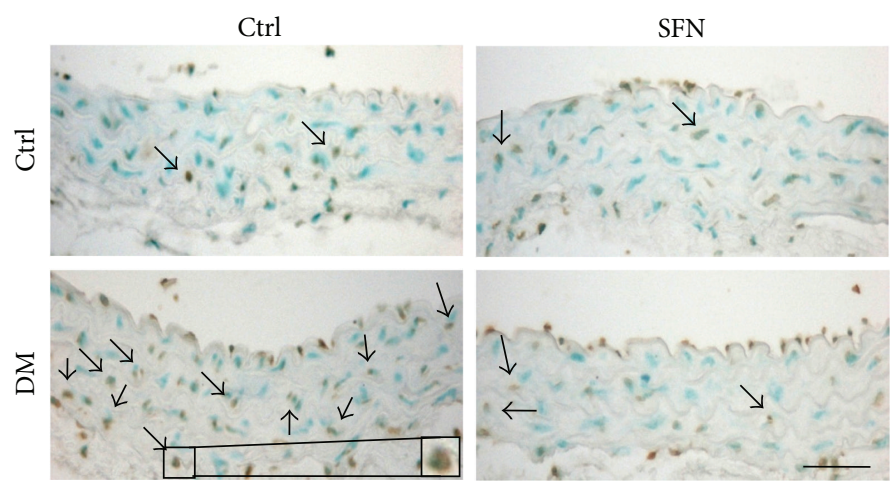

(a)
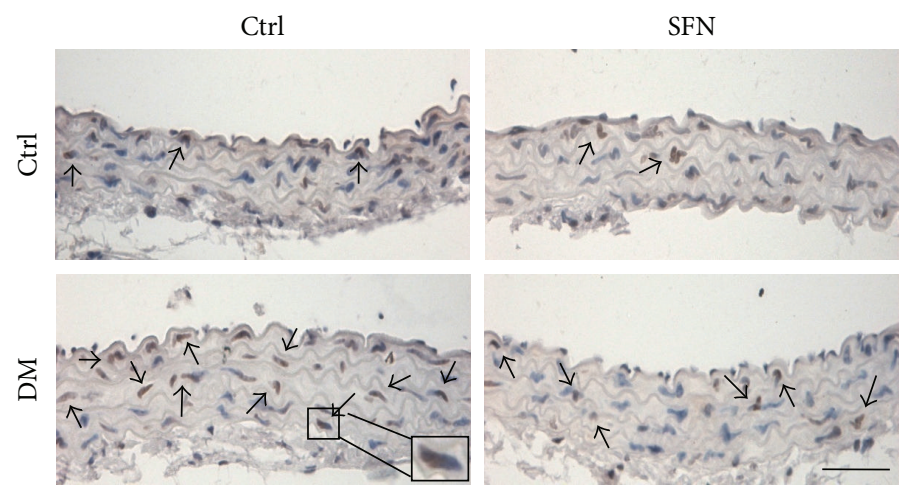

(b)
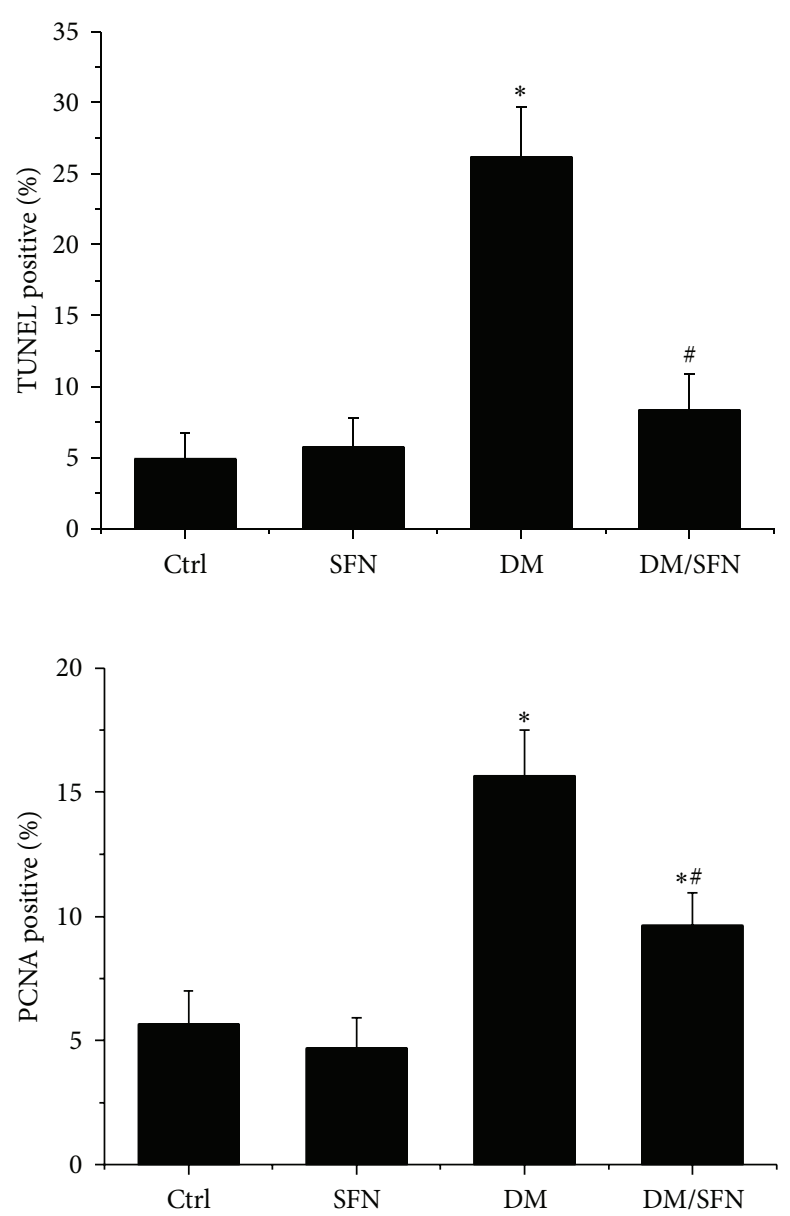

FIGURE 6: Diabetes-induced aortic apoptosis and proliferation increased. The apoptotic cell was examined by TUNEL staining (a) and the proliferation of aortic tunica media was examined by PCNA staining (b), followed by semiquantitative analysis. Data were presented as means $\pm \mathrm{SD}(n=6) .{ }^{*} P<0.05$ versus corresponding Ctrl; ${ }^{\#} P<0.05$ versus corresponding DM. Bar $=50 \mu \mathrm{M}$.

T2DM mice [6]. The next study was conducted to further examine the effect of SFN on the cell death and proliferation by TUNEL staining (Figure 6(a)) and PCNA staining (Figure 6(b)), which showed significant increases of apoptotic cell death and proliferation in the aortas of T2DM mice, but not in the aortas of diabetic mice with SFN administration (DM/SFN group).

3.4. SFN Upregulated the Expression of Nrf2 and Its Downstream Genes. The above results showed that SFN protected diabetic induction of aortic fibrosis, inflammation, and oxidative damage. Considering that oxidative stress has been extensively considered as the pivotal mediator for various cardiovascular complications of diabetic patients, we assume that the above pathological changes in the aortas of T2DM mice may predominantly attribute to the increased oxidative stress. The protective effect of SFN on diabetes-induced aortic pathogenesis may be mediated by upregulation of endogenous antioxidants. SFN is an Nrf2 activator [21], therefore, whether SFN protects the aorta from diabetes by activating Nrf2 was examined first by measuring the expression and transcription of Nrf2. Immunofluorescent staining showed that diabetes significantly decreased Nrf2 protein (Figures 7(a) and 7(b)) expression in the aorta of T2DM compared to control. Similarly, the Nrf2-downstream gene SOD-1 protein (Figures 8(a) and 8(b)) expression also decreased in the aorta of T2DM compared to control. Furthermore, diabetes also significantly downregulated the mRNA expression of Nrf2 (Figure $7(\mathrm{c})$ ) and its downstream antioxidant genes SOD-1 (Figure 8(c)) and HO-1 (Figure 8(d)) in the aorta of T2DM compared to control. Although 4 months of SFN treatment significantly increased $\mathrm{Nrf} 2$ and its downstream antioxidant genes at both protein and mRNA levels in non-DM and T2DM mice.

\section{Discussion}

We have provided the first experimental evidence to show the significant protective effect of SFN on the aorta against T2DM-induced damage. Significant increase of aortic wall thickness, fibrosis, inflammation, oxidative damage, apoptosis and proliferation was developed in type 2 diabetic mice, 
Ctrl
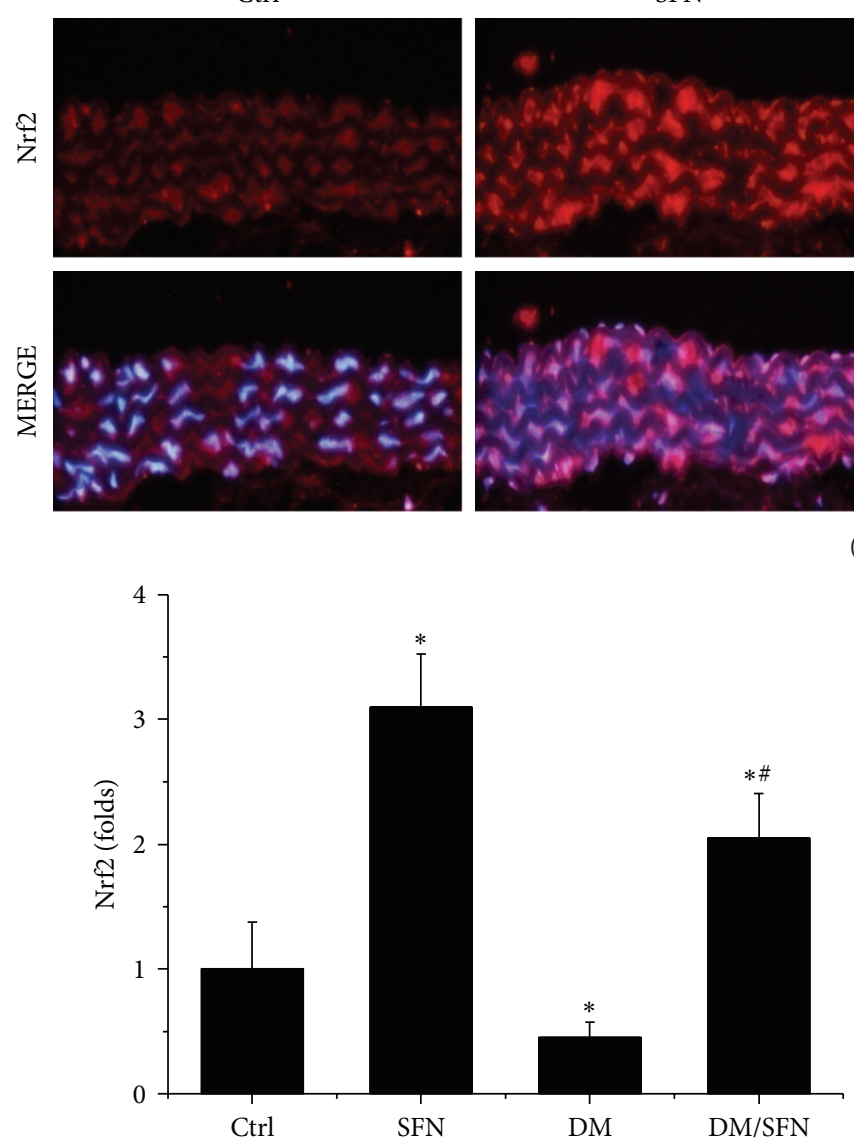

(b)
SFN
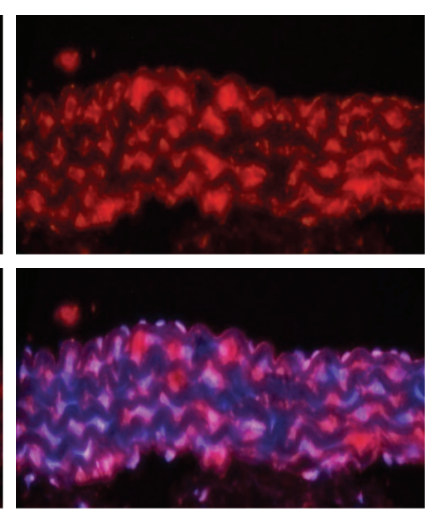

$\mathrm{DM}$
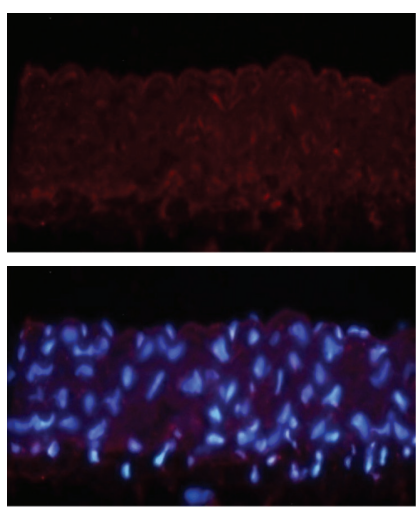

$\mathrm{DM} / \mathrm{SFN}$
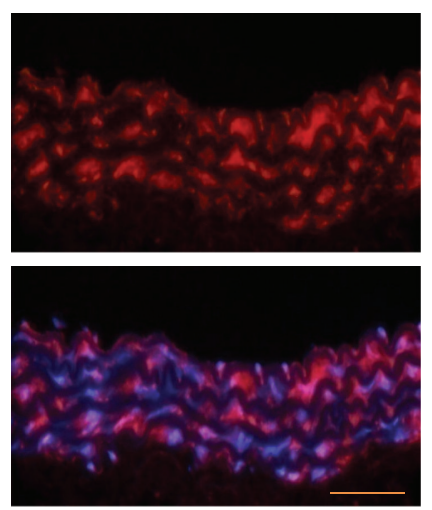

(a)

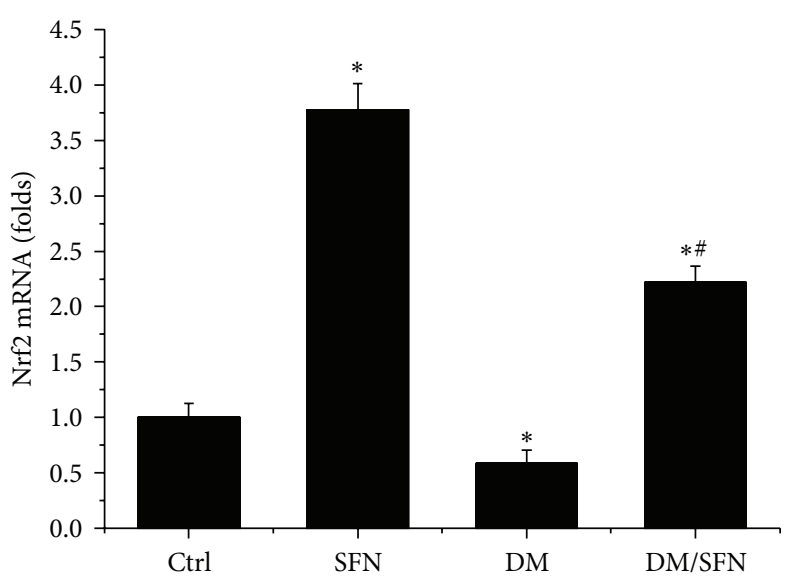

(c)

FIGURE 7: Effects of SFN on aortic expression of Nrf2. Aortic expression of Nrf2 was examined by immunofluorescent staining for its protein expression (red) (a) with semiquantitative analysis (b) and real-time PCR for its mRNA level (c). Data were presented as means \pm SD $(n=6)$. ${ }^{*} P<0.05$ versus corresponding Ctrl; ${ }^{*} P<0.05$ versus corresponding DM. Bar $=50 \mu \mathrm{M}$.

and these pathological changes were significantly prevented by treatment with SFN, which is associated with the upregulation of aortic Nrf2 expression and transcription.

Chronic inflammation plays an important role for the development of various chronic pathogeneses, including diabetes [30-33]. The effects of chronic inflammation include induction of oxidative stress, apoptotic cell death, and abnormal cell proliferation, all of which could contribute to the tissue structural and functional abnormalities [30-33]. In the present study we demonstrated the diabetic induction of aortic inflammation, shown by increased expression of TNF$\alpha$ (Figure 4(a)), VACM-1 (Figure 4(b)) in the aorta of T2DM, which was accompanied with increased aortic oxidative stress (3-NT (Figure 5(a)) and 4-HNE (Figure 5(b))), apoptotic cell death (TUNEL (Figure 6(a))), cell proliferation (PCNA (Figure 6(b))), and remodeling (CTGF (Figure 3(a)) and TGF- $\beta 1$ (Figure 3(b))) in T2DM group. All these pathogenic alterations were prevented by SFN administration. These findings are consistent with the classic concept that inflammation and oxidative stress are reciprocal cause and outcomes [7], both of which are main pathogenic factors for the development of various cardiovascular diseases under stress conditions.
It is known that Nrf2 expression and transcription in vitro and in vivo are increased in response to oxidative stress [34-36]. Ungvari et al. found that HFD increased endothelia ROS levels and endothelial dysfunctions were significantly severer in Nrf2-KO mice than in wild-type mice [37], indicating that adaptive upregulation of Nrf2-driven antioxidant systems effectively attenuates cellular oxidative stress under diabetic conditions [37]. There are a few studies recently, indicating the protective effect by activation of $\mathrm{Nrf} 2$ with various compounds on the aortas under various pathological conditions $[6,38,39]$. Here we have found that SFN treatment also significantly increased Nrf2 expression and function (Figure 7), which indicates that SFN prevents diabetes-induced aortic pathogenesis that may be associated with the upregulation of Nrf2.

In previous studies from our group and others, Nrf2 was found to play a critical role in preventing diabetes-induced aortic damage $[5,6,19]$ and cardiac or renal damage [40-43]. We found that Nrf2 expression in the aorta was significantly upregulated at 3 months without significant aortic damage, but significantly downregulated at 6 months along with significant aortic damage in type 1 diabetic mouse model [5]. 


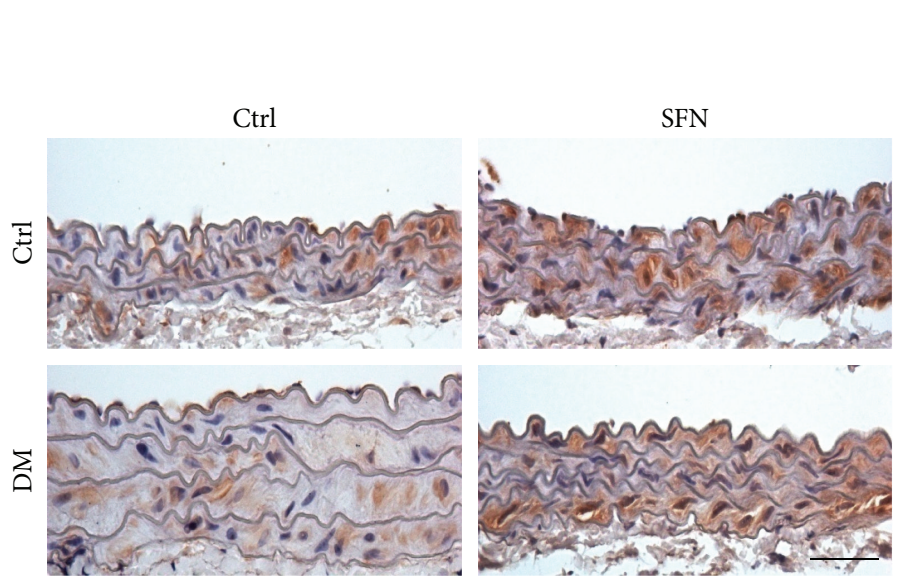

(a)

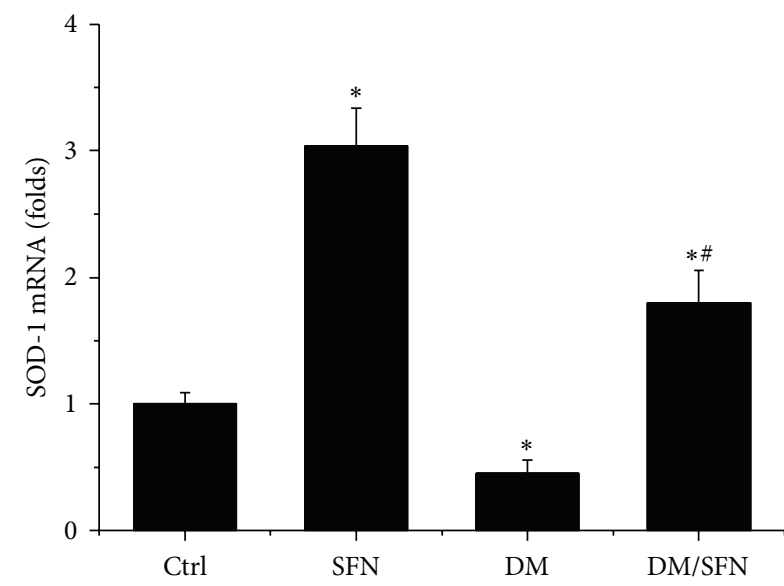

(c)

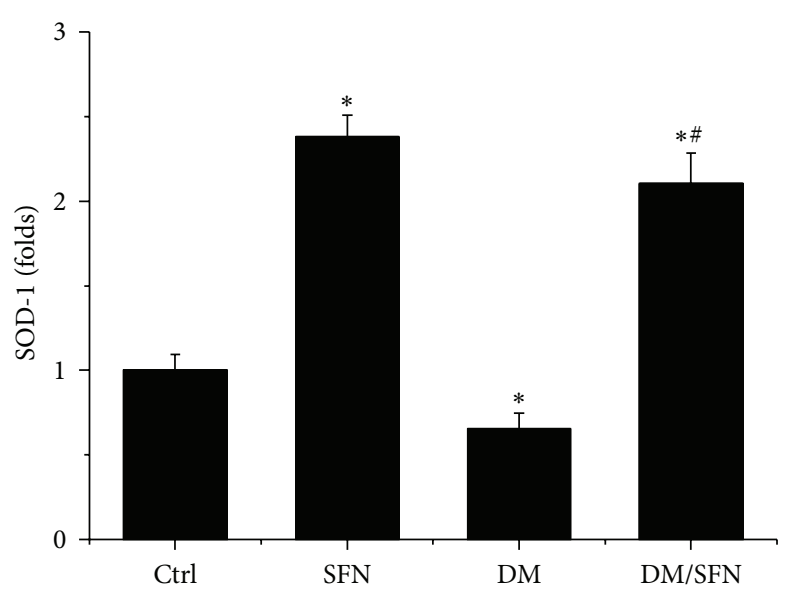

(b)

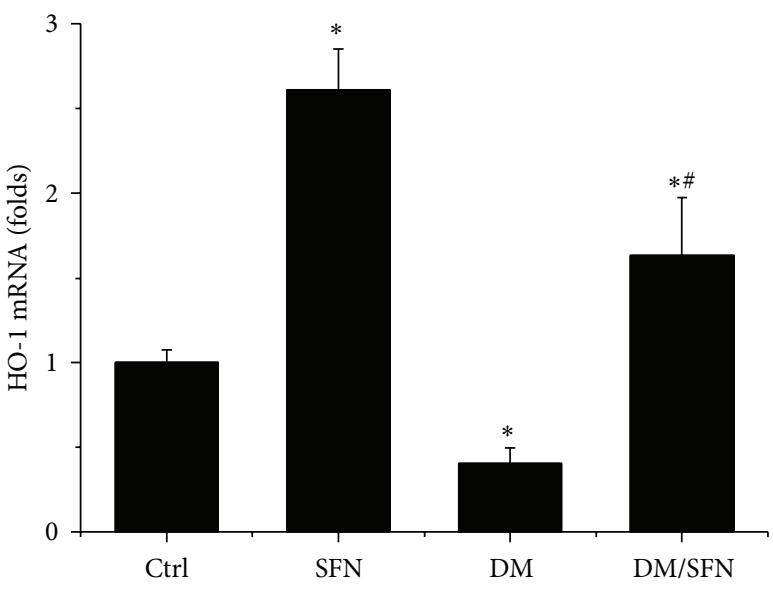

(d)

FIGURE 8: Effects of SFN on aortic expression of Nrf2 downstream genes. Aortic expression of Nrf2 downstream genes SOD-1 expression was examined by immunohistochemical staining for protein expression (a) in aortic tunica media with semiquantitative analysis (b) and real-time PCR at mRNA level SOD-1 (c) and HO-1 (d). Data were presented as means \pm SD $(n=6) .{ }^{*} P<0.05$ versus corresponding Ctrl; ${ }^{\#} P<0.05$ versus corresponding DM. Bar $=50 \mu \mathrm{M}$.

Similarly, aortic expression of Nrf2-driven antioxidant enzymes markedly increases in young mice fed a HFD, but tend to decrease or only mild increase in middle-aged mice fed a HFD, despite the fact that vascular oxidative stress is more severe in HFD-fed middle-aged mice than in young mice [44]. Consistent with these previous studies, we found the significant reduction of Nrf2 expression at both protein and mRNA levels in the aorta of type 2 diabetic mice at 4 months (Figure 7), along with significant downregulation of its transcription function that is reflected by the expression of its downstream antioxidant genes SOD-1 and HO-1 (Figure 8), which were accompanied by significant aortic damage. Most importantly, SFN-treated type 2 diabetic mice showed a significant increase of aortic Nrf2 expression and function. The upregulated Nrf2 and its downstream antioxidant genes in SFN-treated type 2 diabetic mice efficiently reduced diabetes-induced oxidative damage, inflammation, apoptosis, proliferation, and remodeling and eventually significantly prevented the aortic pathological and structural changes.

\section{Conclusions}

In summary, to our knowledge, this is the first study to investigate the protective effects of SFN against T2DM-induced aortic pathological changes. We found that treatment with SFN can completely reverse and/or prevent the progression of diabetes-induced aortic fibrosis, inflammation, oxidative damage, apoptosis, and proliferation in T2DM mice. Mechanism responsible for the preventive effect of SFN is related to upregulation of $\mathrm{Nrf2}$ expression and function to afford potent antioxidant effect. Considering the fact that SFN is a molecule able to be obtained from cruciferous vegetables such as broccoli, cauliflower, or cabbages [24], our findings would be very important for patients with T2DM consider intaking foods rich with SFN for preventing vascular complications.

\section{Conflict of Interests}

There is no conflict of interests to be declared by the authors. 


\section{Authors' Contribution}

Yonggang Wang, Zhigou Zhang, Wanqing Sun, and Yucheng Liu performed the experiments, which were initiated and designed by Jian Sun, Yang Zheng, Quan Liu, and Lu Cai. Lu Cai and Yi Tan were the critical supervisor of the experimental performance and, were critically involved in drafting, writing, and revising the paper. All the authors reviewed the final version of paper.

\section{Acknowledgments}

This study was supported in part by the Basic Research Award from American Diabetes Association (1-11-BA-17, to Lu Cai) and the regular grants from National Natural Science Foundation of China (no. 81273509, to Yi Tan; no. 81370318, to Yang Zheng).

\section{References}

[1] I. D. Atlas, The Global Burden, International Diabetes Federation, Brussels, Belgium, 4th edition, 2009.

[2] A. B. Dart, P. J. Martens, C. Rigatto et al., "Earlier onset of complications in youth with type 2 diabetes," Diabetes Care, 2013.

[3] K. Khavandi, H. Amer, B. Ibrahim et al., "Strategies for preventing type 2 diabetes: an update for clinicians," Therapeutic Advances in Chronic Disease, vol. 4, no. 5, pp. 242-261, 2013.

[4] L. Cai and Y. J. Kang, "Oxidative stress and diabetic cardiomyopathy: a brief review," Cardiovascular Toxicology, vol. 1, no. 3, pp. 181-193, 2001.

[5] X. Miao, Y. Bai, W. Sun et al., "Sulforaphane prevention of diabetes-induced aortic damage was associated with the up-regulation of Nrf2 and its down-stream antioxidants," Nutrition \& Metabolism, vol. 9, no. 1, p. 84, 2012.

[6] X. Miao, Y. Wang, J. Sun et al., "Zinc protects against diabetesinduced pathogenic changes in the aorta: roles of metallothionein and nuclear factor (erythroid-derived 2)-like 2," Cardiovascular Diabetology, vol. 12, p. 54, 2013.

[7] S. Gupta, J. K. Gambhir, O. Kalra et al., "Association of biomarkers of inflammation and oxidative stress with the risk of chronic kidney disease in Type 2 diabetes mellitus in North Indian population," Journal of Diabetes and Its Complications, vol. 27, no. 6, pp. 548-552, 2013.

[8] G. L. King and M. R. Loeken, "Hyperglycemia-induced oxidative stress in diabetic complications," Histochemistry and Cell Biology, vol. 122, no. 4, pp. 333-338, 2004.

[9] I. K. Jeong and G. L. King, "New perspectives on diabetic vascular complications: the loss of endogenous protective factors induced by hyperglycemia," Diabetes \& Metabolism Journal, vol. 35, no. 1, pp. 8-11, 2011.

[10] F. A. Matough, S. B. Budin, Z. A. Hamid, N. Alwahaibi, and J. Mohamed, "The role of oxidative stress and antioxidants in diabetic complications," Sultan Qaboos University Medical Journal, vol. 12, no. 1, pp. 556-569, 2012.

[11] L. Cai, "Diabetic cardiomyopathy and its prevention by metallothionein: experimental evidence, possible mechanisms and clinical implications," Current Medicinal Chemistry, vol. 14, no. 20, pp. 2193-2203, 2007.

[12] J.-M. Lee and J. A. Johnson, "An important role of Nrf2-ARE pathway in the cellular defense mechanism," Journal of Biochemistry and Molecular Biology, vol. 37, no. 2, pp. 139-143, 2004.
[13] V. O. Tkachev, E. B. Menshchikova, and N. K. Zenkov, "Mechanism of the Nrf2/Keap1/ARE signaling system," Biochemistry, vol. 76, no. 4, pp. 407-422, 2011.

[14] M. McMahon, K. Itoh, M. Yamamoto, and J. D. Hayes, "Keap1dependent proteasomal degradation of transcription factor Nrf2 contributes to the negative regulation of antioxidant response element-driven gene expression," Journal of Biological Chemistry, vol. 278, no. 24, pp. 21592-21600, 2003.

[15] R. K. Thimmulappa, K. H. Mai, S. Srisuma, T. W. Kensler, M. Yamamoto, and S. Biswal, "Identification of Nrf2-regulated genes induced by the chemopreventive agent sulforaphane by oligonucleotide microarray," Cancer Research, vol. 62, no. 18, pp. 5196-5203, 2002.

[16] W. Li and A.-N. Kong, "Molecular mechanisms of Nrf2mediated antioxidant response," Molecular Carcinogenesis, vol. 48, no. 2, pp. 91-104, 2009.

[17] G. P. Sykiotis, I. G. Habeos, A. V. Samuelson, and D. Bohmann, "The role of the antioxidant and longevity-promoting Nrf2 pathway in metabolic regulation," Current Opinion in Clinical Nutrition and Metabolic Care, vol. 14, no. 1, pp. 41-48, 2011.

[18] E. L. Donovan, J. M. McCord, D. J. Reuland et al., "Phytochemical activation of $\mathrm{Nrf} 2$ protects human coronary artery endothelial cells against an oxidative challenge," Oxidative Medicine and Cellular Longevity, vol. 2012, Article ID 132931, 9 pages, 2012.

[19] X. Miao, W. Cui, W. Sun et al., "Therapeutic effect of MG132 on the aortic oxidative damage and inflammatory response in OVE26 type 1 diabetic mice," Oxidative Medicine and Cellular Longevity, vol. 2013, Article ID 879516, 12 pages, 2013.

[20] A. T. Dinkova-Kostova, W. D. Holtzclaw, R. N. Cole et al., "Direct evidence that sulfhydryl groups of Keap1 are the sensors regulating induction of phase 2 enzymes that protect against carcinogens and oxidants," Proceedings of the National Academy of Sciences of the United States of America, vol. 99, no. 18, pp. 11908-11913, 2002.

[21] Y.-S. Keum, "Regulation of the Keap1/Nrf2 system by chemopreventive sulforaphane: implications of posttranslational modifications," Annals of the New York Academy of Sciences, vol. 1229, no. 1, pp. 184-189, 2011.

[22] C. E. Guerrero-Beltrán, M. Calderón-Oliver, J. PedrazaChaverri, and Y. I. Chirino, "Protective effect of sulforaphane against oxidative stress: recent advances," Experimental and Toxicologic Pathology, vol. 64, no. 5, pp. 503-508, 2012.

[23] P. C. Evans, "The influence of sulforaphane on vascular health and its relevance to nutritional approaches to prevent cardiovascular disease," EPMA Journal, vol. 2, no. 1, pp. 9-14, 2011.

[24] J. W. Fahey and P. Talalay, "Antioxidant functions of sulforaphane: a potent inducer of phase II detoxication enzymes," Food and Chemical Toxicology, vol. 37, no. 9-10, pp. 973-979, 1999.

[25] J. Mu, J. Woods, Y.-P. Zhou et al., "Chronic inhibition of dipeptidyl peptidase- 4 with a sitagliptin analog preserves pancreatic $\beta$-cell mass and function in a rodent model of type 2 diabetes," Diabetes, vol. 55, no. 6, pp. 1695-1704, 2006.

[26] Y. Zhao, Y. Tan, S. Xi et al., "A novel mechanism by which SDFlbeta protects cardiac cells from palmitate-induced endoplasmic reticulum stress and apoptosis via CXCR7 and AMPK/p38 MAPK-mediated interleukin-6 generation," Diabetes, vol. 62, no. 7, pp. 2545-2558, 2013.

[27] Y. Bai, W. Cui, Y. Xin et al., "Prevention by sulforaphane of diabetic cardiomyopathy is associated with up-regulation of Nrf2 expression and transcription activation," Journal of Molecular and Cellular Cardiology, vol. 57C, pp. 82-95, 2013. 
[28] A. Qazi, J. Pal, M. Maitah et al., "Anticancer activity of a broccoli derivative, sulforaphane, in barrett adenocarcinoma: potential use in chemoprevention and as adjuvant in chemotherapy," Translational Oncology, vol. 3, no. 6, pp. 389-399, 2010.

[29] K. Mahéo, F. Morel, S. Langouët et al., "Inhibition of cytochromes P-450 and induction of glutathione S- transferases by sulforaphane in primary human and rat hepatocytes," Cancer Research, vol. 57, no. 17, pp. 3649-3652, 1997.

[30] D. I. Simon, "Inflammation and vascular injury: basic discovery to drug development," Circulation Journal, vol. 76, no. 8, pp. 1811-1818, 2012.

[31] N. Ishigami, K. Isoda, T. Adachi et al., "Deficiency of CuZn superoxide dismutase promotes inflammation and alters medial structure following vascular injury," Journal of Atherosclerosis and Thrombosis, vol. 18, no. 11, pp. 1009-1017, 2011.

[32] V. O. Puntmann, P. C. Taylor, and M. Mayr, "Coupling vascular and myocardial inflammatory injury into a common phenotype of cardiovascular dysfunction: systemic inflammation and aging-a mini-review," Gerontology, vol. 57, no. 4, pp. 295-303, 2011.

[33] C. Davis, J. Fischer, K. Ley, and I. J. Sarembock, "The role of inflammation in vascular injury and repair," Journal of Thrombosis and Haemostasis, vol. 1, no. 8, pp. 1699-1709, 2003.

[34] P. Palsamy and S. Subramanian, "Resveratrol protects diabetic kidney by attenuating hyperglycemia-mediated oxidative stress and renal inflammatory cytokines via Nrf2-Keap1 signaling," Biochimica et Biophysica Acta, vol. 1812, no. 7, pp. 719-731, 2011.

[35] Y. Tan, T. Ichikawa, J. Li et al., "Diabetic downregulation of Nrf2 activity via ERK contributes to oxidative stress-induced insulin resistance in cardiac cells in vitro and in vivo," Diabetes, vol. 60, no. 2, pp. 625-633, 2011.

[36] Y.-C. Yang, C.-K. Lii, A.-H. Lin et al., "Induction of glutathione synthesis and heme oxygenase 1 by the flavonoids butein and phloretin is mediated through the ERK/Nrf2 pathway and protects against oxidative stress," Free Radical Biology and Medicine, vol. 51, no. 11, pp. 2073-2081, 2011.

[37] Z. Ungvari, L. Bailey-Downs, T. Gautam et al., "Adaptive induction of NF-E2-related factor-2-driven antioxidant genes in endothelial cells in response to hyperglycemia," American Journal of Physiology-Heart and Circulatory Physiology, vol. 300, no. 4, pp. H1133-H1140, 2011.

[38] A. Ishikado, K. Morino, Y. Nishio et al., "4-Hydroxy hexenal derived from docosahexaenoic acid protects endothelial cells via Nrf2 activation," PloS One, vol. 8, no. 7, Article ID e69415, 2013.

[39] P. R. Avila, S. O. Marques, T. F. Luciano et al., "Resveratrol and fish oil reduce catecholamine-induced mortality in obese rats: role of oxidative stress in the myocardium and aorta," The British Journal of Nutrition, vol. 110, no. 9, pp. 1580-1590, 2013.

[40] X. He, H. Kan, L. Cai, and Q. Ma, "Nrf2 is critical in defense against high glucose-induced oxidative damage in cardiomyocytes," Journal of Molecular and Cellular Cardiology, vol. 46, no. 1, pp. 47-58, 2009.

[41] T. Jiang, Z. Huang, Y. Lin, Z. Zhang, D. Fang, and D. D. Zhang, "The protective role of $\mathrm{Nrf} 2$ in streptozotocin-induced diabetic nephropathy," Diabetes, vol. 59, no. 4, pp. 850-860, 2010.

[42] J. B. De Haan, "Nrf2 activators as attractive therapeutics for diabetic nephropathy," Diabetes, vol. 60, no. 11, pp. 2683-2684, 2011.

[43] W. Cui, B. Li, Y. Bai et al., "Potential role for Nrf2 activation in the therapeutic effect of MG132 on diabetic nephropathy in
OVE26 diabetic mice," American Journal of Physiology, vol. 304, no. 1, pp. E87-E99, 2013.

[44] A. R. Collins, C. J. Lyon, X. Xia et al., "Age-accelerated atherosclerosis correlates with failure to upregulate antioxidant genes," Circulation Research, vol. 104, no. 6, pp. e42-e54, 2009. 


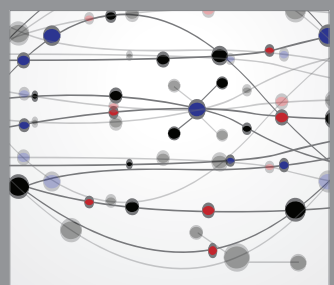

The Scientific World Journal
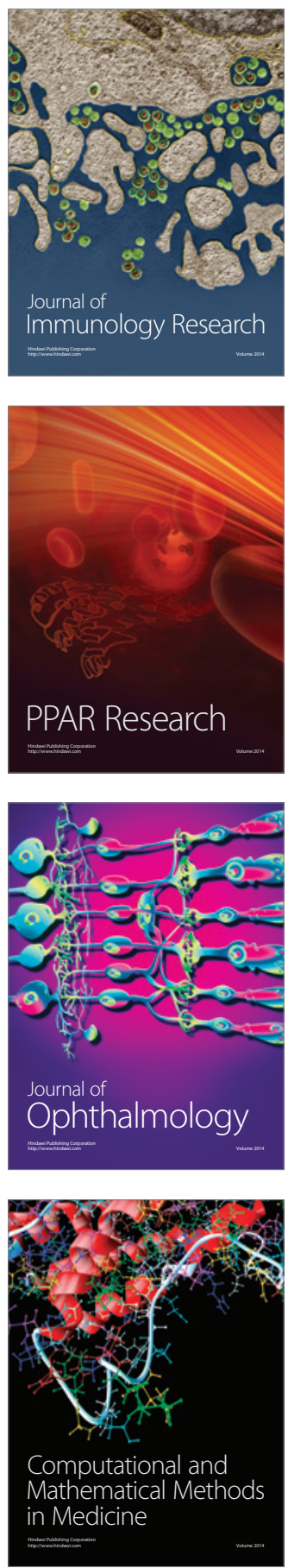

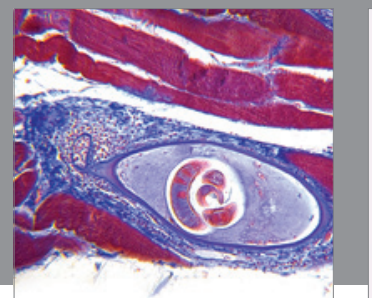

Gastroenterology

Research and Practice
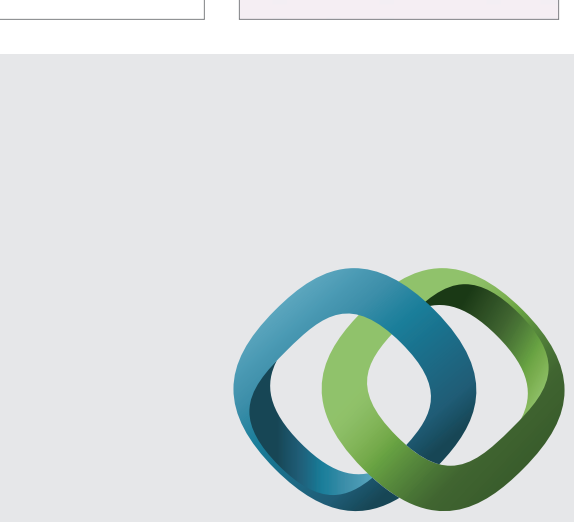

\section{Hindawi}

Submit your manuscripts at

http://www.hindawi.com
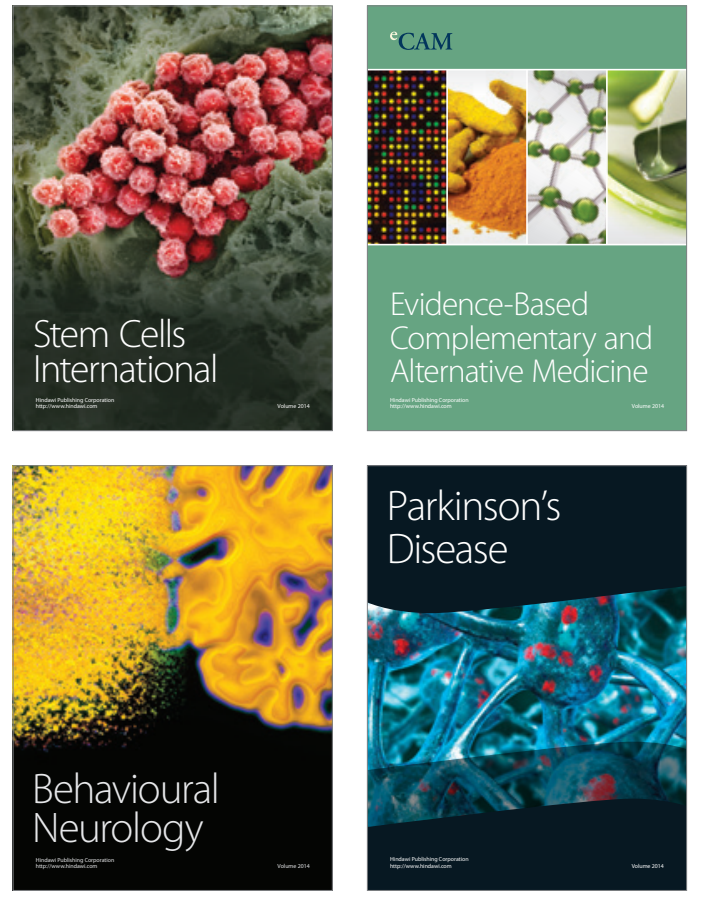
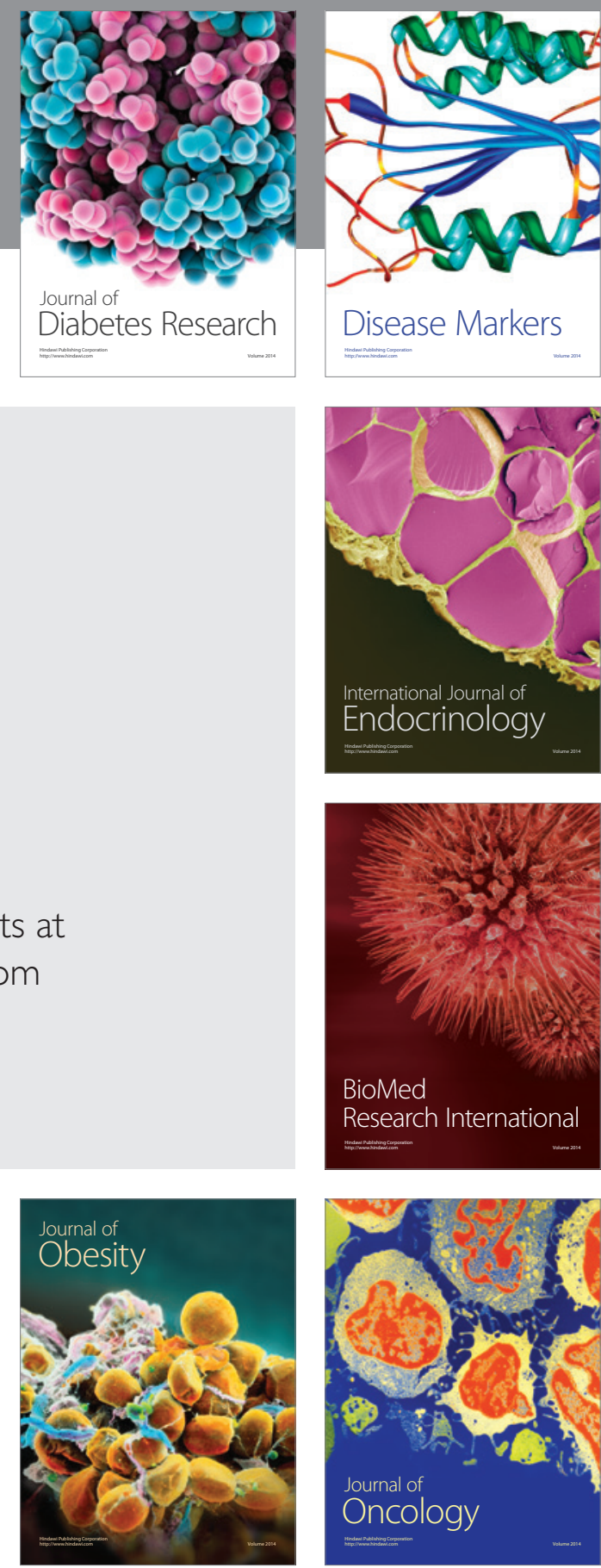

Disease Markers
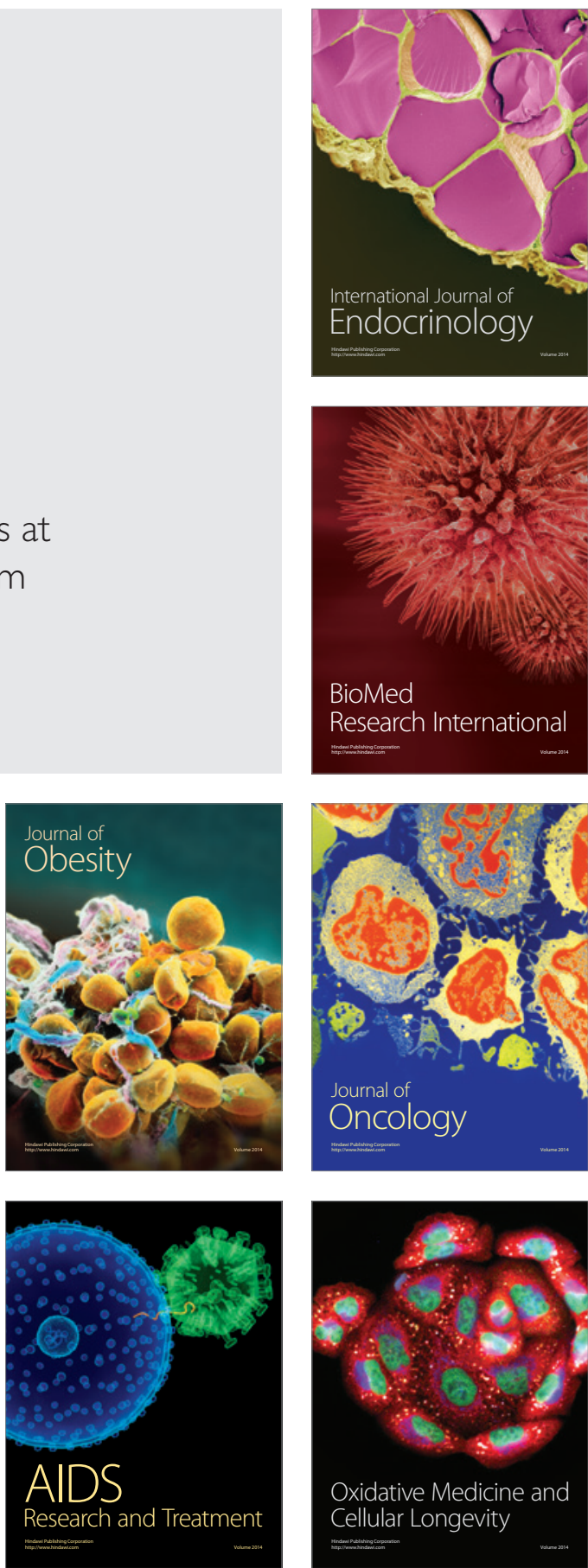